\title{
Realidades invisibilizadas: pobreza e impacto de la crisis a partir de una investigación feminista en el área metropolitana de Barcelona
}

\author{
Laura SALES GUTIÉRREZ, \\ Fundació Surt. Fundació de Dones. Fundació Privada. \\ laura.sales@surt.org
}

Recibido: 08-03-2013

Aceptado: 06-06-2013

\begin{abstract}
RESUMEN
El artículo plantea una crítica feminista al análisis de la pobreza y la crisis económica, a partir de una investigación cuantitativa y cualitativa realizada en 2009 con 2.609 mujeres del área metropolitana de barcelona. se parte de un análisis crítico del concepto de pobreza, que cuestiona los métodos convencionales de cálculo de la tasa de pobreza, y de diversos aspectos invisibilizados del impacto de la crisis económica en las mujeres, en términos de desempleo y de calidad del desempleo, reprecarización del trabajo femenino, y aumento del trabajo reproductivo. Estos impactos se muestran a partir de estadísticas de empleo y las conclusiones del estudio realizado por la fundació surt.
\end{abstract}

Palabras clave: análisis feminista, crisis, pobreza, mujeres

\section{Invisibilised realities: poverty and impact of the crisis on women a feminist research study in the Barcelona metropolitan area}

\begin{abstract}
This article sets out a critical feminist analysis of poverty and the economic crisis, based a quantitative qualitative research carried out in 2009 with 2,609 women in the Barcelona metropolitan area. it begins with a critical analysis of the concept of poverty, which challenges conventional methods of calculating the poverty rate. it also refers to several aspects of the invisible differential impact of the economic crisis on women, in terms of women's unemployment and its quality, and a process of recasualization of women's work and increased obligations in care work. These impacts are shown with employment statistics and the conclusion of the research carried out by the surt foundation.
\end{abstract}

Keywords: feminist analysis, crisis, poverty, women

\section{REFERENCIA NORMALIZADA}

Sales Gutiérrez, L. (2013). Realidades invisibilizadas: pobreza e impacto de la crisis a partir de una investigación feminista en el área metropolitana de Barcelona. Cuadernos de Relaciones Laborales Vol, 30, núm.2, p. $435-451$.

SUMARIO: Introducción 1. La pobreza desde el análisis feminista. 2. El sesgo androcéntrico en el análisis de la crisis. Invisibilidades conceptuales y estadísticas. 2.1. Realidades invisibles, impactos invisibles. 2.2. Aumento del trabajo reproductivo 3. conclusiones. 4. Bibliografía. 


\section{Introducción}

El análisis crítico del concepto de pobreza y el uso, también crítico, del término "nueva pobreza" en este monográfico invitan a un replanteamiento de conceptos asentados en las Ciencias Sociales. En esta tarea de revisión de prácticas y conceptos hegemónicos, el análisis feminista cuenta con una larga historia: una de las tareas continuadas de los estudios feministas ha sido la visibilización de que el conocimiento se genera desde las perspectivas y los comportamientos socialmente dominantes, que han sido los masculinos. En el ámbito de los estudios feministas, esta crítica dio lugar al concepto de androcentrismo, acuñado por la escritora estadounidense Charlotte Perkins Gilman a principios del siglo XX para describir el proceso por el cual la masculinidad se constituía en el centro, la norma y la medida del mundo. Desde este planteamiento, por una parte, la masculinidad se establece como normativa y todo lo que queda fuera de la masculinidad queda excluido. Por otra parte, los comportamientos y los puntos de vista masculinos se identifican con lo universal, mientras que los femeninos se consideran una desviación de esta pauta (Perkins Gilman, 1911). A partir de este concepto, la epistemología feminista ha elaborado un ingente cuerpo teórico (Harding, 1986).

En el ámbito que nos ocupa, el aspecto más relevante es que, como consecuencia de esta perspectiva androcéntrica, en el conocimiento en general y en las Ciencias Sociales y Económicas en particular, la teoría ha sido durante mucho tiempo ciega a las diferencias de género. La economía feminista ha hecho un ejercicio de deconstrucción de la teoría económica hegemónica (Carrasco, 2006) y acuñado conceptos que permiten visibilizar y analizar realidades hasta ahora ocultas: las experiencias y perspectivas de las mujeres. Sin embargo, los análisis feministas siguen estando en los márgenes de la teoría económica, como ha quedado ampliamente demostrado en el contexto de la crisis económica actual.

Esta ceguera conceptual desemboca en una recogida parcial de los datos socioeconómicos, que conduce a un análisis de la realidad social también parcial y prejuiciado, y de este modo se retroalimentan las invisibilidades. Para referirse a este fenómeno, la economista feminista Cristina Carrasco acuñó el feliz término "estadísticas bajo sospecha" (Carrasco, 2007).

De esta invisibilidad y esta ausencia surgió precisamente el estudio Dones en crisi ("Mujeres en crisis"), en el que se basa este artículo, realizado por la Fundació Surt a partir del análisis cuantitativo y cualitativo de 2.609 mujeres residentes en la ciudad de Barcelona y su área metropolitana atendidas por la organización a lo largo de 2008 y 2009. La experiencia de los y las profesionales y las entidades que realizan atención social con mujeres desde diversos ámbitos son fundamentales para entender la realidad multidimensional de la "nueva pobreza", pues son quienes desde primera línea se enfrentan diariamente a las necesidades y las capacidades de las personas y su constante adaptación a las circunstancias cambiantes en la última fase del capitalismo.

La Fundació Surt es una fundación de mujeres que trabaja desde 1993 en la ciudad de Barcelona para la inserción laboral de las mujeres desde una perspectiva 
integral, constructivista y de género. En torno a este propósito, la entidad despliega multitud de iniciativas, desde la intervención comunitaria a la atención individual en casos de violencia machista, talleres de empoderamiento, formación para profesionales, planes de igualdad y estudios de investigación que permiten desarrollar nuevos planteamientos para adaptar la práctica profesional de atención a las mujeres a los nuevos retos sociales.

El estudio Dones en crisi partió de la inquietud de las profesionales de atención directa a mujeres de la entidad ante la percepción de que los medios de comunicación transmitían una visión de la crisis económica con un claro sesgo androcéntrico. La invisibilidad de los impactos de la recesión en las mujeres no coincidía con la experiencia de la Fundació Surt en la atención directa de la creciente demanda femenina para la participación en programas de inserción laboral. Ya en esta primera fase de la crisis económica, la visión sesgada reflejada en los medios hacía pensar que dicha invisibilidad pudiera desembocar en políticas de recuperación de la crisis que no afrontaran adecuadamente las problemáticas de las mujeres e incluso pudieran agravar las desigualdades de género preexistentes.

En este contexto, el objetivo general del proyecto Dones en crisi fue analizar el impacto de la crisis económica actual sobre la situación laboral y familiar de las mujeres, a fin de contar con información que permitiera, por una parte, la visibilización de la dimensión de género de la crisis ante la opinión pública y, por otro, el diseño de intervenciones adecuadas para el fomento del empleo femenino desde los dispositivos de inserción laboral existentes y las administraciones públicas.

Tras la realización de este estudio, en una primera fase de la crisis económica y su impacto en el mercado laboral, otros muchos han destacado los múltiples impactos de la crisis sobre las mujeres (Gálvez y Torres, 2010; Carrasco, 2012; Larrañaga, 2009; De Villota, 2011), en una tendencia agudizada por la posterior deriva neoliberal que ha reducido a su mínima expresión las políticas sociales y de igualdad en España.

Estos estudios coinciden en la insuficiencia de los actuales datos macroeconómicos para explicar los impactos multidimensionales de la crisis económica sobre las mujeres, y en concreto los impactos en el ámbito del trabajo reproductivo realizado en los hogares y en la calidad de vida de las mujeres. No hay que olvidar que la crisis económica es una crisis del capitalismo global que se suma a crisis preexistentes, como la crisis de los cuidados, la "dimensión olvidada de la crisis actual" (Carrasco, 2009).

\section{La pobreza desde el análisis feminista}

La crisis económica global ha provocado un preocupante aumento de la pobreza, tradicionalmente feminizada. Según los últimos datos de Eurostat, correspondientes a 2011, la tasa de riesgo a la pobreza femenina en Catalunya es del 26,8\% (el 30\% en España), 1,1 puntos por encima de la masculina. 
Recientemente (Belzunegui, 2012) se ha cuestionado la adecuación del término feminización de la pobreza como realidad descriptiva del fenómeno. En efecto, el término "feminización" parece indicar el aumento de la brecha entre la tasa de pobreza femenina y la masculina. Sin embargo, los datos aportados por Belzunegui et al a partir del Panel de Hogares de la Unión Europa y de la Encuesta de Condiciones de Vida no demuestran esta progresión.

\section{Gráfico 1. Tasa de pobreza relativa ( $60 \%$ de la renta mediana) por sexo (porcentajes). España, 1996-2010}

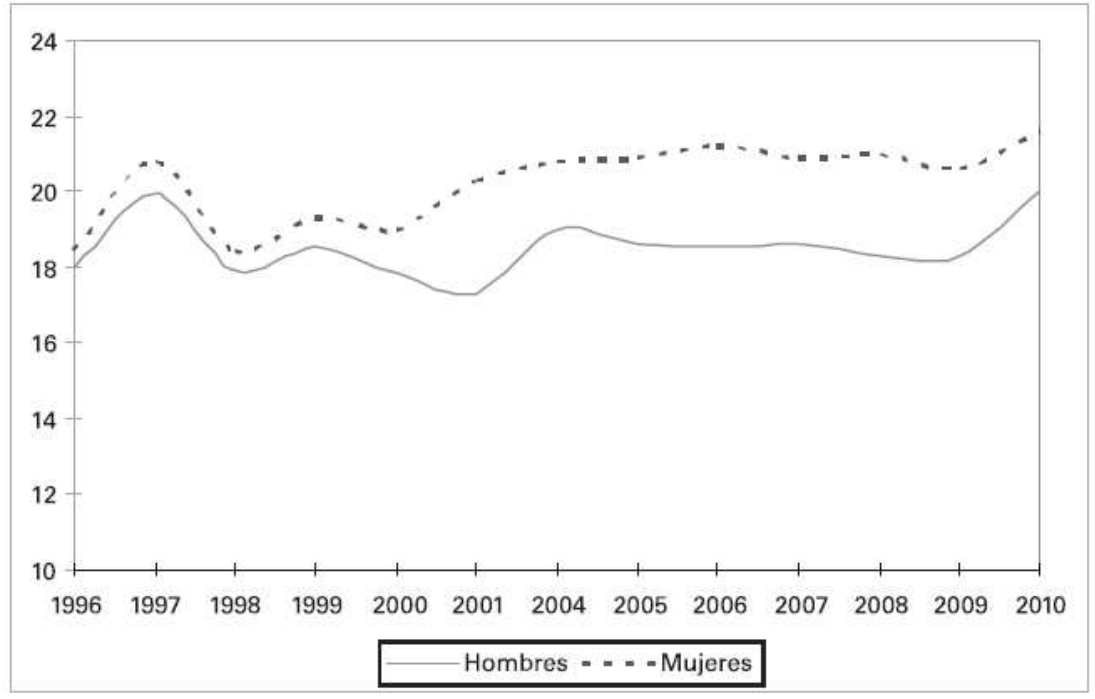

Fuente: Elaboración propia a partir de PHOGUE (1996-2001) y ECV (2004-2010).

Fig. 1. Fuente: Belzunegui, Ángel (coord.) (2012). Socialización de la pobreza en España. Barcelona: Icaria, p. 73

De acuerdo con estos datos, si bien la tasa de pobreza relativa $(60 \%$ de la renta mediana) de las mujeres es ligeramente superior a la de los hombres, no es cierto que el diferencial entre los sexos haya aumentado en tiempos de crisis. Por todo ello, los autores proponen el término "socialización de la pobreza" como alternativa al de feminización.

El término "feminización de la pobreza" se ha puesto también en cuestión desde otra perspectiva: su falta de atención a las diferencias de renta existentes entre las mujeres. Más allá del análisis económico, la visibilización simbólica de los distintos ejes de desigualdad y no sólo el de género ha sido otra de las reivindicaciones del feminismo global de las últimas décadas, a partir de los feminismos negros y poscoloniales y la teoría de la interseccionalidad (Mohanty, 1988; Crenshaw, 1991). Desde los márgenes de los feminismos, se ha criticado el uso de la categoría mujer no sólo por su invisibilización de las diferencias entre mujeres, sino porque en la 
práctica se identifica con "mujer blanca, occidental, heterosexual y de clase media". La tasa de pobreza relativa es una clara ilustración de estas diferencias entre mujeres: los datos de la ECV y el PHOGUE muestran una mayor vulnerabilidad de las mujeres con familias monoparentales, por ejemplo, que el término "feminización" invisibiliza.

Sin embargo, cabe preguntarse si el análisis exclusivo de los ingresos refleja adecuadamente el alcance y las características de la pobreza de las mujeres, así como las diferencias existentes entre mujeres diversas. Con objeto de realizar un análisis más profundo y multidimensional de las condiciones de vida, se han propuesto otros métodos alternativos de medición de la pobreza. Uno de ellos es el propuesto por Francesc Valls y recogido en la misma monografía de Belzunegui et al. La propuesta parte del cuestionamiento del hogar como medida de ingresos, puesto que presupone una distribución equitativa de los ingresos dentro de la unidad familiar o de convivencia, con lo que se invisibiliza la dimensión institucional de la familia en el orden sexo/género.

Este análisis alternativo de la pobreza por razón de género calcula la tasa de pobreza bajo el supuesto de autonomía. Tal cálculo de la tasa de pobreza de cada persona mayor de 15 años que no estuviera estudiando ni tuviera que depender solamente de sus ingresos arroja unos resultados muy distintos al cálculo convencional y, en particular, una mayor gravedad de la pobreza femenina. Por ello, Belzunegui et al concluyen que "los métodos convencionales de cálculo de la pobreza tienden a ocultar una parte esencial de la pobreza femenina" (Valls, 2012).

Igualmente esencial es tener en cuenta para el análisis de la pobreza cálculos que van más allá de la pobreza monetaria. Belzunegui et al examinan 36 variables pertinentes a cinco dimensiones de análisis relativas a la inclusión social para obtener "una radiografía más detallada de las situaciones de vulnerabilidad". Todas ellas muestran que "las condiciones de vida de las mujeres son más desfavorables que las de los hombres" e inciden especialmente en una peor percepción del estado de la propia salud y una menor capacidad de consumo básico.

\section{El sesgo androcéntrico en el análisis de la crisis. Invisibilidades conceptuales y estadísticas}

Las carencias conceptuales y estadísticas que invisibilizan la naturaleza de la pobreza femenina son extensibles al análisis académico, mediático y político de la crisis económica. Desde que, en 2008, el mercado laboral global empezó a tambalearse, las mujeres nunca han sido identificadas socialmente como afectadas directas por la destrucción de puestos de trabajo. Ciertamente, en el momento de estallido de la crisis, el paro afectó principalmente a sectores masculinizados (como la construcción o la automoción), con lo que se invirtió la tendencia de un desempleo femenino superior al masculino, pero el desempleo creció rápidamente en el sector servicios, altamente feminizado. Según datos de la EPA del cuarto trimestre de 
2012, la tasa de paro femenino en Catalunya es del $23,2 \%$ y la masculina, del $24,8 \%$, una diferencia de 1 punto que no justifica esta invisibilidad.

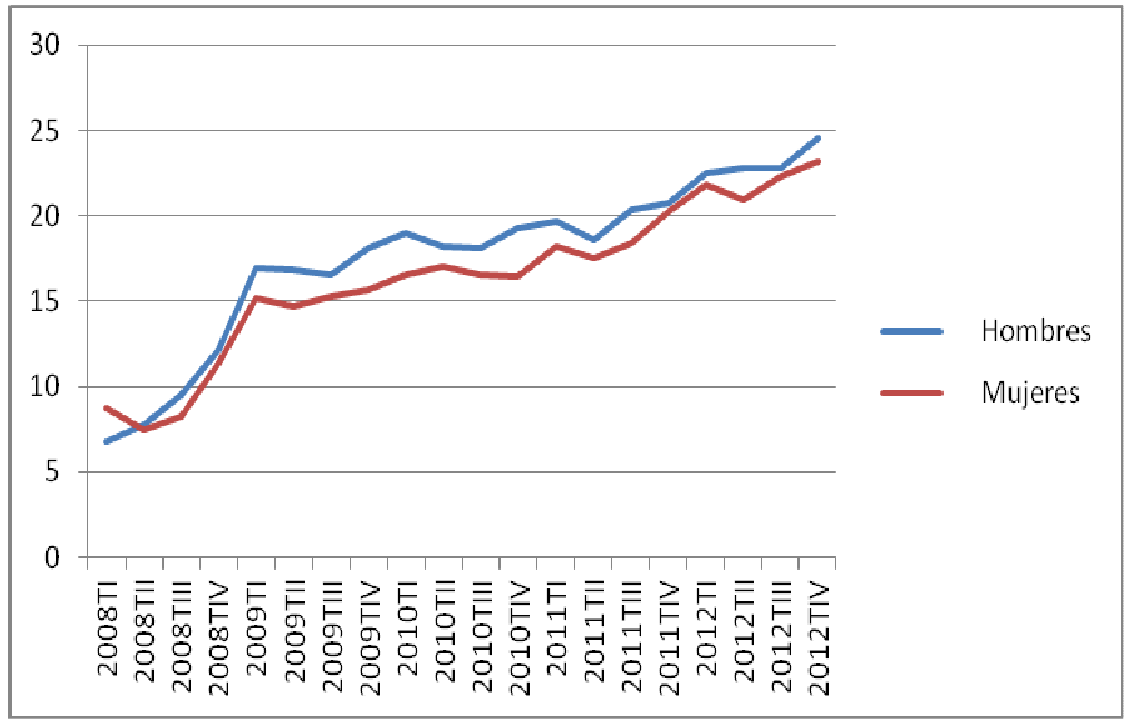

Fig. 2. Tasa de paro (2008-2012)

Fuente: Idescat, a partir de datos de la encuesta de población activa del INE

La tasa de paro femenina en Catalunya ha aumentado en periodo de crisis, pero el incremento ha sido más moderado que el del paro masculino y se ha producido en periodos diferentes. El paro masculino comenzó a subir en el primer trimestre de 2008, afectado por la destrucción de empleo en sectores muy masculinizados, y ha ido aumentando de forma continuada hasta 2012. En cambio, el paro femenino comenzó a crecer también en el primer trimestre de 2008, pero se recuperó el siguiente trimestre, momento a partir del cual hubo más hombres parados que mujeres. Esta leve superioridad del paro masculino es un fenómeno que no se producía desde hace décadas, cuando la tasa de actividad de las mujeres en Cataluña era mucho menor, y que no ha llegado a producirse en el conjunto del estado español, donde el paro femenino todavía supera el masculino.

Este diferencial en el empleo no puede ocultar el hecho estructural de que antes de la agudización de la destrucción de empleo la tasa de paro femenino era superior a la masculina. Si a esta desigualdad se añaden todos los elementos de precariedad del trabajo femenino (parcialidad, salarios más bajos, informalidad, etc.), es evidente que la conservación de un puesto de trabajo femenino no implica necesariamente una situación de ventaja en el mercado laboral. La reducción del diferencial en el paro masculino y femenino no se puede interpretar como un logro de la igualdad en el mercado de trabajo, sino como un impacto inmediato de la crisis en el empleo masculino. 
Por otra parte, a partir de mediados de 2011 se produjo en Catalunya un aumento del desempleo femenino (asociado principalmente a la destrucción del empleo en el sector servicios) y una moderación del paro masculino, un fenómeno que desde el análisis económico feminista se ha teorizado con la denominada "hipótesis de protección" o "amortiguadora" del desempleo por parte de las mujeres (Rubery, 1993; Larrañaga, 2011).

Otro factor que suele pasar inadvertido es el aumento de la tasa de actividad femenina, que ha tenido un incremento sostenido durante el período de crisis, siguiendo la tendencia previa, mientras que la actividad masculina ha descendido. En el contexto de la crisis, algunos estudios han vinculado el aumento de la actividad laboral femenina a una incorporación al empleo de mujeres previamente "inactivas" en el mercado (ADECCO, 2009), mientras que el descenso de la actividad masculina se ha relacionado con el llamado "efecto del trabajador desanimado".

Pero la pérdida de empleo y el aumento de la actividad son sólo parte de la fotografía. Indicadores clave de desigualdad, como la brecha salarial o la tasa de parcialidad, se han mantenido estables durante la crisis, lo que demuestra la pervivencia de la desigualdad de partida entre hombres y mujeres. La encuesta de estructura salarial de 2010 muestra que la diferencia en términos de salario entre mujeres y hombres se ha mantenido invariable durante los últimos años y se sitúa en Catalunya el $16,9 \%$ (por debajo del $22,5 \%$ en el conjunto del estado). Otro indicador fundamental es la tasa de parcialidad: las mujeres ocupadas trabajan semanalmente en Catalunya una media de 30,6 horas, mientras que los hombres trabajan 36,1 horas (de acuerdo con datos de la EPA del cuarto trimestre de 2012).

Son estas desigualdades estructurales las que no se han tenido en cuenta en la interpretación de la destrucción de empleo en sectores feminizados, equiparando la calidad del puesto de trabajo masculino en el lugar de trabajo femenino (con mayor temporalidad, parcialidad de jornada y menor salario). Si la calidad del empleo es peor en el caso de las mujeres, también lo es la calidad del desempleo: no es lo mismo perder un puesto de trabajo estable, a jornada completa - caso típicamente masculino--, que perder un trabajo temporal, a jornada parcial y con un salario inferior, que son las características habituales del empleo femenino. Por lo tanto, la conservación del puesto de trabajo no supone para las mujeres disponer de ingresos suficientes para su autonomía.

\subsection{Realidades invisibles, impactos invisibles}

Precisamente factores como la calidad del empleo y del desempleo quedan muchas veces ocultos por las estadísticas, que de este modo impiden una comprensión amplia y matizada la realidad. Las estadísticas tampoco han permitido visibilizar suficientemente la pérdida de empleo en sectores feminizados, como el del cuidado, dada la extensión de la economía sumergida en estos sectores. No se pueden obviar los efectos de pérdida de empleo en cadena en estos ámbitos, muy evidente en el caso del servicio doméstico.

Por último, y fundamentalmente, el contacto con las mujeres atendidas en las entidades sociales muestra un proceso de reprecarización del trabajo femenino — con 
peores condiciones laborales, más temporalidad y salarios inferiores--, que profundiza las desigualdades estructurales de género en el mercado de trabajo y repercute en una ocupación aún más insuficiente para la autonomía de las mujeres. Si bien el empeoramiento de las condiciones de trabajo ha afectado a toda la población y es una tendencia agravada por las dos últimas reformas laborales en España, la situación de partida desigual de las mujeres en el mercado de trabajo las ha desplazado hacia una precariedad insostenible.

Por todo ello, en el marco del estudio Dones en crisi se pretendió visibilizar esta realidad semioculta con una investigación cualitativa y cuantitativa realizada con mujeres activas del área metropolitana de Barcelona atendidas en la Fundación SURT entre enero de 2008 y septiembre de 2009.

La base de datos de las mujeres atendidas por la Fundación Surt permite recoger datos estadísticos relevantes para el análisis de la situación laboral y familiar de las mujeres, con variables como el país de procedencia, la edad, la nacionalidad, la situación de convivencia, las responsabilidades familiares, las prestaciones percibidas, el nivel formativo, la situación laboral, el tiempo en el paro y la experiencia al mercado laboral formal e informal. Son variables diseñadas en función de las experiencias de las mujeres y de los factores que inciden en su inserción laboral efectiva en el mercado. Por ello, el análisis de los datos de 2.609 mujeres y la realización de actividades de investigación cualitativa con 14 mujeres ( 9 entrevistas en profundidad y un grupo de discusión) permitió recoger una imagen de conjunto de los efectos de la crisis en diferentes esferas de la vida de las mujeres.

Los resultados de la investigación cualitativa, en especial, demostraron que la crisis había supuesto una gran transformación para las mujeres, que percibían claramente un antes y un después respecto a la recesión en ámbitos tan diversos como la situación laboral, el nivel de ingresos o la relación de pareja. Cabe destacar, especialmente, el impacto causado no sólo directamente por la falta de empleo y el descenso de los ingresos, sino por el efecto en cadena provocado por el deterioro de la situación económica de otras personas, que repercutía en aspectos muy diversos de la vida de las mujeres, generaba el aumento de las deudas, la precarización de la vivienda o la reducción de la pensión por separación. Las mujeres entrevistadas lo expresaban de este modo:

D5: "Lo que pasa es que, a ver, yo pensé que iba a pagar porque, claro, yo contaba con un dinero que yo había prestado, ¿sabes?, 3.000 euros. Entonces yo dejé la Seguridad Social así, en el tiempo, que se acumule. [...] Pero, claro, a la que le había prestado el dinero me quedó mal y se fue a otro lado y me quedé...". (...) "Bueno, ahora con esto de que vino de golpe la crisis, también lo golpeó a él y ahora mismo también está mal. Hombre, él si pudiera me pasara más, ¿sabes?, pero claro, como él también vive aparte, paga habitación en otro lugar...".

D8: "Ha encontrado un paquistaní, que le ha dicho 'Mira, tengo un piso, no sé qué, está bueno, para vosotras, está bien'. Y no quiero ni adelanto, de ni..., bueno, ni fianza, bueno, contrato, cosas así, nada. 600 euros al mes. (...) Y nos hemos enterado por los vecinos que aquel piso no es del paquistaní, que estamos viviendo de ocupas." 
En el caso de las mujeres inmigradas, se observaba un impacto con características diferenciadas en los casos de mujeres con responsabilidades familiares transnacionales. Este impacto transnacional de la crisis en el país de acogida se asocia a una disminución de las remesas enviadas a las familias residentes en el país de origen y el consiguiente efecto en su bienestar, así como con el aumento de la presión sobre las mujeres por no poder sostener adecuadamente a las personas a su cargo.

D3: "Ya no como antes, que mandaba los 300 euros cada mes. Ahora mando lo justo. Cosa que mis hijos del colegio que estaban en un colegio pagado, ahora están en un colegio del gobierno, que no se paga. (...) [En mi país] tengo mi familia. Mi papá también. Mi madre no tengo, pero... Le ayudo cuando puedo, también. Mira, ahora estuvo enfermo, tuve que hacerle dos giros. (...) Él depende de mí. (...) Claro, tengo hermanos varones, pero no tienen ellos. La única que... No tengo recursos, pero, bueno, la única que podría ayudarle soy yo. (...) Tuve que ir yo, coger prestado...”

D5: "Reducir incluso lo que más me produjo fue mandar a mi familia. Eso también fatal. Porque ellos, claro, contaban... Yo ayudaba a dos hermanos y a mi padre. Hoy puedo ayudar a mi padre. (...) Tengo una hermana también allá que está separada, ¿sabes? Lo pasa mal. Le puse un psicólogo porque ella es más sensible, ¿sabes? Le sentó fatal. Un psicólogo y eso también tuve que reducir. Y a veces me da pena porque quisiera ayudarla un poquito más."

En el ámbito del empleo, el estudio mostraba una situación laboral afectada por la crisis en distintos niveles. Por una parte, se apreciaba un gran aumento del número de mujeres activas interesadas en iniciar un proceso de inserción laboral, con cifras que se doblaban respecto al periodo anterior (fig. 3). Los picos de atención en meses concretos del período corresponden a la apertura de programas de inserción en la entidad.

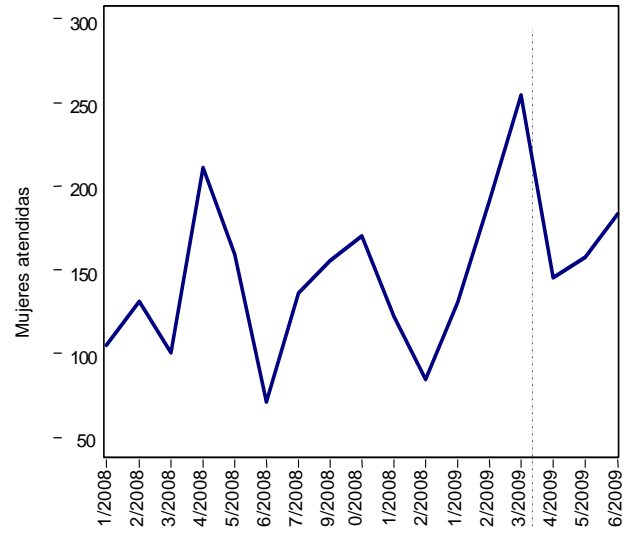

Fig. 3. Sesiones informativas de la Fundació Surt por meses (enero de 2008 a junio de 2009) 
En segundo lugar, en el período analizado se detectó también una importante diversificación de los perfiles de mujeres en procesos de inserción laboral, con un aumento de la proporción de mujeres autóctonas (leve, del 4\%, pero que invierte la tendencia en la organización en los últimos años) y, especialmente, el descenso en la proporción de mujeres sin estudios y el aumento del porcentaje de mujeres con estudios superiores atendidas en la organización (fig. 4).

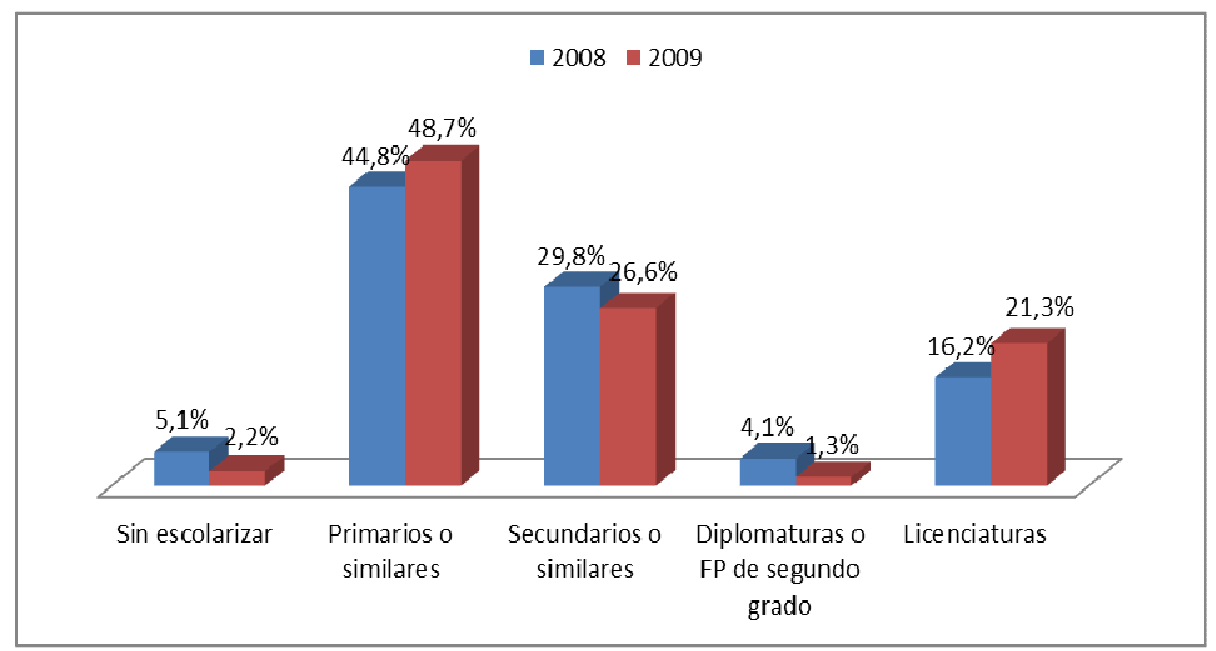

Fig. 4. Mujeres atendidas entre enero de 2008 y junio de 2009, por nivel de estudios y año de acogida.

Respecto al desempleo, se recogieron también aspectos destacables, como el hecho de que el desempleo de mayor duración aparecía asociado fundamentalmente a las mujeres con responsabilidades familiares (fig. 5).

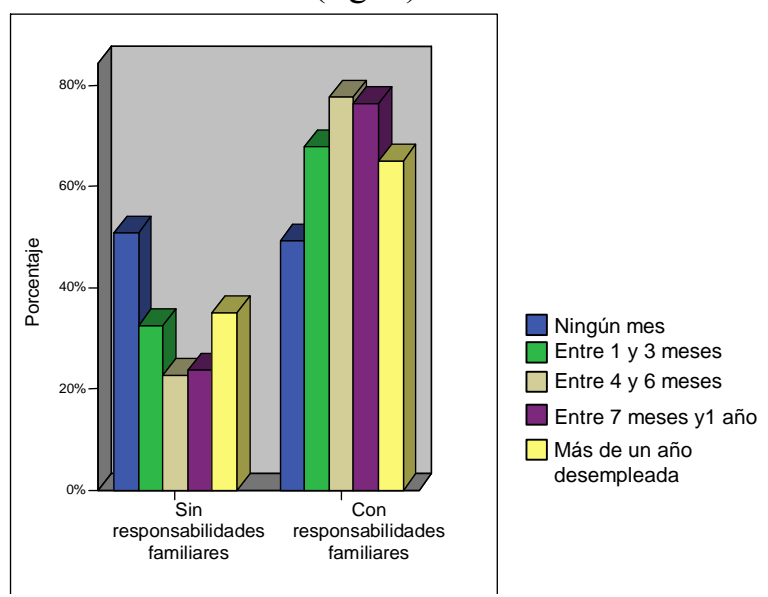

Fig. 5. Mujeres desocupadas por tiempo de desempleo y responsabilidades familiares 
Especialmente relevante es que muchas de las mujeres entrevistadas vivían en periodo de crisis una situación que se podría definir como "desempleo a tiempo parcial". No habían dejado de trabajar totalmente, pero se han visto forzadas a reducir las horas trabajadas en empleos que previamente ya eran parciales y precarias, y por tanto insuficientes para su autonomía. La finalización de las relaciones laborales de las mujeres no se había producido exclusivamente por despido, sino también por baja voluntaria (especialmente en los casos de las que trabajaban en el mercado informal), por la percepción de un descenso de la demanda en su sector. De este modo, las mujeres entrevistadas describieron un descenso de la demanda en sectores feminizados, muchos de ellos desarrollados principalmente en el ámbito informal e invisibles a las estadísticas, como el trabajo sexual y los servicios en el hogar y de atención a las personas, pero también en la hostelería o el sector editorial.

D4: "Mucha gente que trabajé con ellos ya se ingresaba en, cómo se llama, residencias, porque ya no podía pagar a alguien que lo cuidara. (...) Tenía unas casas de limpiar. Vale, combiné el trabajo sexual con las horas que tenía de hacerlo. Y después ya me paré porque ya empezó la crisis y, imagínate, la gente ya no tenía tanto dinero de pagar. Porque pagaban unos 8, 10 euros a la hora. $Y$ hay gente que no te puede pagar. Y para una hora no te conviene irte con el metro, ida y vuelta, ¿me entiendes?"

G5: "La señora con la crisis traspasó el restaurante, porque ya no le iba gente."

G2: "Y pegué para ir a limpiar casas. No recibía llamadas. (...) Nada, nada. Totalmente parado. Nunca recibí una llamada."

El deterioro de la calidad del empleo y el aumento de la precariedad también quedaron recogidos en las conclusiones del estudio. Las mujeres entrevistadas relataban cómo la crisis había supuesto un deterioro de la calidad del trabajo y un aumento del empleo precario, en relación a una bajada de salarios, un trabajo temporal e inestable, y un aumento de la carga de trabajo.

D6: "Y también, que todos me decían lo mismo, hay crisis, mientras aguantemos los que estamos, aunque tengamos que trabajar el triple, aguantaremos, más que contratar a otra persona."

Otro aspecto remarcable reflejado en el estudio fue lo que se denominó "paro sumergido". Las cifras oficiales de paro registrado ocultan el número de mujeres en proceso de búsqueda de empleo que no constan oficialmente como desempleadas. En estrecha relación con el amplio concepto de trabajo empleado en el estudio, el concepto de paro utilizado comprendía también todo el desempleo femenino, independientemente de su registro en las oficinas de trabajo de la Generalitat de Catalunya. El acceso a la base de datos de usuarias de la Fundació Surt permitía medir la extensión de este fenómeno y analizar los factores que podrían incidir en la ausencia de las mujeres activas en los registros oficiales. Los resultados del estudio mostraron que el 19,2\% de las mujeres desempleadas no estaban registradas en las 
oficinas de empleo de la Generalitat de Catalunya y por tanto constaban en el registro oficial como mujeres "inactivas". Sin embargo, eran mujeres que habían emprendido una búsqueda activa de empleo, como evidencia el hecho de su atención a un dispositivo de inserción laboral como la Fundació Surt, y que difícilmente se podían considerar inactivas, dado el alto porcentaje de mujeres con responsabilidades familiares: el 50\% de las autóctonas y el 54,8\% de las inmigradas.

Se observó también que las mujeres en paro sumergido eran mayoritariamente mujeres inmigradas $(84 \%)$. Cabe destacar que la regularización de su situación administrativa no implicaba necesariamente un registro en el paro, ya que el $73,8 \%$ de las mujeres inmigradas no registradas tenían permiso de residencia y trabajo (fig. 6).
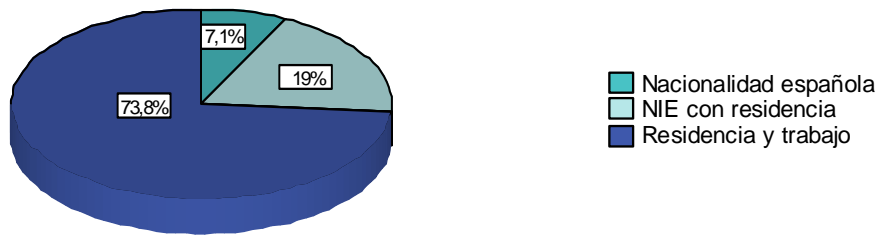

Fig. 6. Mujeres inmigradas en paro sumergido, según su situación legal

El análisis de las trayectorias laborales de las mujeres en paro sumergido permitió arrojar luz sobre una posible razón por la que estas mujeres no se registran como demandantes de empleo (fig. 7): a lo largo de su trayectoria laboral, encuentran empleo por lo menos en alguna ocasión en el mercado informal (96,2\%). Un 68\% de estas mujeres supuestamente inactivas había trabajado siempre sin contrato y el $32 \%$ se había movido entre el mercado formal e informal.

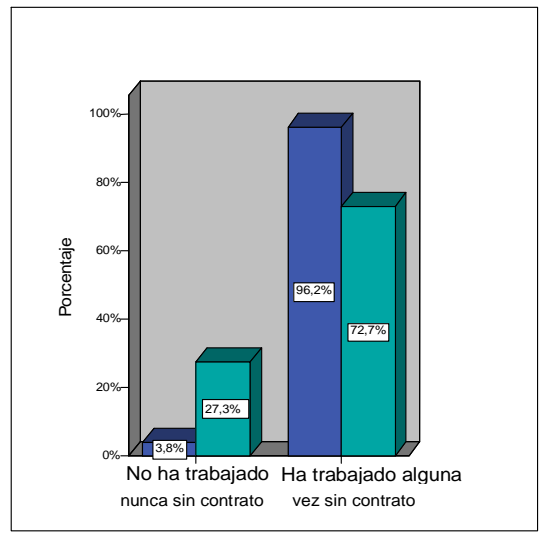

No registrada como demandante de empleo

Registrada como demandante de empleo

Fig. 7. Mujeres desempleadas, experiencia laboral informal y registro como demandantes de empleo. 


\subsection{Aumento del trabajo reproductivo}

A la situación laboral descrita hay que añadir la desigualdad de género más grande y más invisible en el trabajo, que es la carga de trabajo reproductivo: el trabajo doméstico y de cuidado de personas que las mujeres realizan sin remuneración en el invisible espacio doméstico es un trabajo con una exigua valoración social, aunque, como recuerda la economista Cristina Carrasco, garantiza la sostenibilidad de la vida (2009).

$\mathrm{Y}$ esta desigualdad también se ha profundizado en tiempos de crisis, con un aumento de la carga de trabajo como consecuencia de la reducción de gastos en el hogar para hacer frente a la situación económica. Las mujeres, como principales gestoras de la economía doméstica, son las que han encarado la reestructuración de prioridades en tiempos de vacas flacas y las que han reunido a la familia para comunicar que había que prescindir de pequeños lujos. Son las mujeres quienes siguen pasando más tiempo comparando precios entre tiendas buscando la mejor oferta y las que sufren en primera persona el estrés y la ansiedad derivados de la incapacidad de proveer a hijos e hijas de mejores condiciones de vida.

En el estudio Dones en crisi emergieron múltiples estrategias para reducir los gastos en el hogar y, asociado a estas estrategias, una intensificación del trabajo de cuidado de las mujeres. Por una parte, en periodo de crisis la economía familiar se veía afectada por numerosas transformaciones en relación a los hábitos de consumo, como consecuencia de las estrategias de reducción de gastos puestas en marcha para hacer frente a la bajada de ingresos. Destacaba, por ejemplo, el control del consumo en actividades de ocio, especialmente en restaurantes. Este cambio había comportado para las mujeres un aumento de su carga de trabajo doméstico en la cocina y un incremento de la reclusión en el hogar. En algunos casos, se relataba un consumo desbordado previo a la crisis y se defendía la moderación del consumo como valor moral.

Por otra parte, la investigación cualitativa realizada mostraba que la pérdida de ingresos y las estrategias de reducción de gastos de vivienda habían supuesto en algunos casos la reorganización de los hogares, con alteraciones importantes en los miembros de la unidad de convivencia que subvierten la organización familiar tradicional, como ilustran las siguientes citas:

D6: "Arreglé una habitación en mi casa y la estoy alquilando."

G4: "Y me tocó mandar a mi madre y a mi hija para Colombia."

D8: "Mi marido está en la cárcel. Por eso mismo comenzó. (...)Bueno, estaba con mi amiga.

En este sentido, el análisis cuantitativo de los perfiles de las mujeres atendidas mostró una mayoría de mujeres que vivían solas y en familias nucleares, pero es destacable la evolución entre los años 2008 y 2009, con un descenso de los hogares unifamiliares y un aumento de hogares con más de dos miembros, que podría indi- 
car una estrategia para hacer frente a la pérdida de ingresos en situación de crisis económica (fig.8).

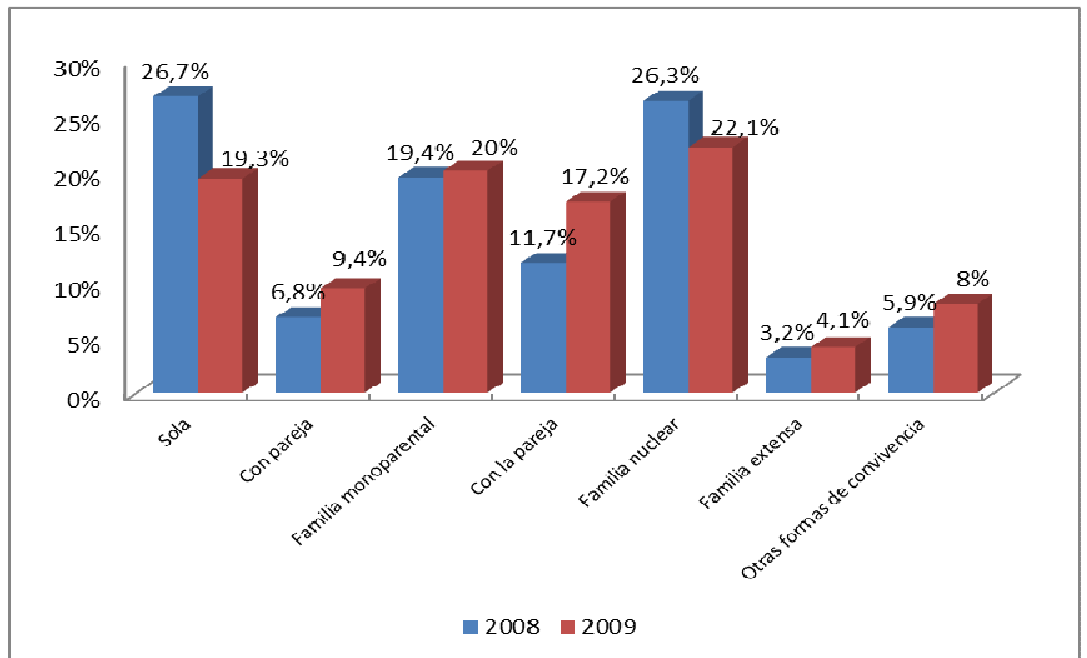

Fig. 8. Mujeres atendidas entre enero de 2008 y junio de 2009, por situación de convivencia y año de acogida.

Otro de los resultados destacables del estudio fue la visibilización del papel central de las mujeres como impulsoras y gestoras de la nueva economía familiar en tiempo de crisis. Las mujeres son mayoritariamente las administradoras del hogar y por tanto las encargadas de decidir las estrategias adecuadas para hacer frente al descenso de los ingresos. Este trabajo creativo y de organización de los miembros del hogar implicaba, en tiempos de crisis, un aumento de las horas de trabajo de gestión y de la responsabilidad de las mujeres, que debían hacer una supervisión de las tareas de los demás miembros del hogar, incapaces de realizarlas de forma autónoma.

D9: "Yo administro, porque él compraría igual todo y yo le digo que hay que comprar algo más barato para poder luego comprar un zumo o Danone para el niño."

G5: "Es que, es que siempre la mujer organiza mejor la comida y como que alcanza mejor. Porque yo les dejo algunas veces que vayan al mercado. Y cuando vuelvo no... Se ha ido el dinero y lo que hay... Para tres días."

En estrecha relación con esto, el estudio detectaba también un aumento de la carga de trabajo doméstico y familiar como consecuencia de las estrategias para hacer frente a la crisis, sin que existieran, por otro lado, cambios significativos en el reparto de tareas que aligerasen la carga de trabajo de las mujeres. La reducción de los gastos en restaurantes y en otros servicios del hogar y de atención a las personas 
conllevaba un aumento de la carga de trabajo reproductivo en la gran mayoría de los hogares de las mujeres entrevistadas.

D4: "Ya se nota, ya no me voy tanto a pasear, ni comer en restaurantes."

D7: "Ahora comemos cada día en casa."

Este incremento de la carga de trabajo era asumido por las mujeres, que siguen siendo las principales responsables del trabajo de cuidado. En segundo término, las personas que asumían más responsabilidades eran otras mujeres de la familia y amistades, tanto residentes en el hogar como externas, con o sin remuneración. Como consecuencia, si antes de la recesión la doble presencia (en el mercado de trabajo y la gestión del hogar) resultaba agotadora para las mujeres, los malabarismos imprescindibles para economizar en tiempos de crisis y la presión de la situación de urgencia volvían la situación insostenible.

D2: “A ver, él con los niños se pone muy nervioso, no tiene aguante. (...) [Y en las tareas de la casa] eso sí que me ayuda. No mucho, pero bueno, algo me hace."

D1: "Siempre mi madre, es la comida, que esté siempre preparada la comida, y de los niños también. Nos turnamos."

D3: "Bueno, yo he tenido siempre la garantía de que mi marido tiene a su familia aquí. Entonces como tiene dos sobrinas."

D5: "Yo tengo que pagar chica y a veces no me llega."

Por último, el estudio detectó un empeoramiento del bienestar psicosocial de las mujeres. Muchas de las mujeres entrevistadas expresaban un malestar físico y psicológico alimentado por la angustia de la gestión cotidiana de la precariedad, con trastornos psicosomáticos (alteraciones del sueño, pérdida del apetito, ahogos), estrés y depresión, e incluso cierta tensión en las relaciones de pareja y con los y las familiares.

D3: "Yo creo que la cabeza me va, me va a explotar de tantas cosas. Yo digo tan jo ven y tanta responsabilidad que tengo."

D5: “ ¡Son muchas cosas! Y todo esto intento llevarlo... Espero no estresarme no más. Porque tú sabes que llega un momento en que tú ya no puedes. (...) O sea, desde lo que yo tengo la preocupación, desde las deudas, yo ya esto de comer ya... Con el estrés... (...) Me he adelgazado mucho."

D8: "De dormir, bueno. Se agudizan los pensamientos..."

D7: "De salud estoy bien. (...) Pero yo me noto una ansiedad que antes no la tenía." D3: "A veces estoy irritable yo o está irritable él. Por la mínima cosa a veces discutimos. Pero entiendo que él se siente impotente, yo también, de no tener..." 


\section{Conclusiones}

La investigación cuantitativa y cualitativa realizada en la Fundació Surt en 2009 pone sobre la mesa varios impactos de la crisis económica sobre las mujeres que han pasado desapercibidos ante la opinión pública. El desempleo en sectores feminizados, la reprecarización del trabajo femenino y el aumento del trabajo reproductivo son algunos de los efectos sobre el empleo y las condiciones de vida de las mujeres producidos en el contexto de crisis.

Sin embargo, la situación descrita en el apartado anterior corresponde a los inicios de la recesión, antes de que se pusieran en marcha las políticas de abordaje de la crisis. Está por ver si las medidas tomadas por la gestión de la crisis, y especialmente por el mantenimiento y la promoción del empleo, impulsadas en muchos casos por la urgencia de la situación, podrían haber empeorado aún más las desigualdades estructurales que afectan al empleo femenino.

En un contexto en que la brújula del interés político y mediático raramente se ha desplazado de la creación de empleo en sectores tradicionalmente masculinizados, las medidas puestas en marcha por las administraciones públicas con el objetivo de abordar la crisis no sólo no habrían tenido un efecto correctivo sobre la situación económica, sino que en gran medida podrían agravar la desigualdad de género, al tiempo que se moderaba la inversión en políticas de igualdad (Larrañaga, 2011). En esta misma línea podrían entenderse los impactos de las políticas de ajustes en servicios públicos y la desatención de las políticas sociales. Pero es precisamente ahora que las políticas de igualdad de género son más necesarias que nunca para construir un nuevo modelo productivo y social que atienda realmente los derechos y las necesidades de todas las personas.

\section{Bibliografía}

ADECCO y Fundación ADECCO (2009): IV Informe. Perfil da la mujer trabajadora, Madrid.

Belzunegui, Ángel (coord.) (2012). Socialización de la pobreza en España. Barcelona: Icaria.

Carrasco, C. (2009). Mujeres, sostenibilidad y deuda social. En: Revista de Educación, número extraordinario 2009. Madrid: Ministerio de Educación, Política Social y Deporte.

Carrasco, C. et al (2007). Estadístiques sota sospita. Proposta de nous indicadors des de l'experiència femenina. Barcelona: Institut Català de les Dones. 
Carrasco, Cristina (2006). La economía feminista: una apuesta por otra economía. Disponible en: http://www.seminariovirtual.com.ar/seminario20102/documentos/Carrasco-recomendadoAlmaEspino.pdf

Carrasco, Cristina (2012). No es una crisis, es el sistema, Con la A, n. ${ }^{\circ}$ 1, 8 de marzo de 2012.

Crenshaw, Kimberlé (1991). Mapping the Margins: Intersectionality, Identity Politics, and Violence against Women of Color. Stanford Law Review 43, 1241-1279.

De Villota, Paloma (2011). El impacto de la crisis económica en los tributos y en la sostenibilidad del nuevo modelo social en España. Aproximación desde una perspectiva de género, Investigaciones Feministas, vol 2, 71-94.

Gálvez Muñoz, Lina, y Torres López, Juan (2010). Desiguales: Mujeres y Hombres ante la crisis financera. Barcelona, Icaria.

Harding, Sandra (1986) The Science Question i Feminism. Milton Keynes: Open University Press.

Larrañaga Sarriegi, Mertxe (2009), Mujeres, tiempos, crisis: combinaciones variades, Revista de Economía Crítica, ${ }^{\circ}$ 8, segundo semestre 2009

Larrañaga, Mertxe, Jubeto, Yolanda y De la Cal, Ma Luz. (2011). Tiempos de crisis, tiempos de des-ajustes, tiempos precarios, tiempos de mujeres, Investigaciones Feministas, vol 2, 95-111.

Mohanty, Chandra Talpade. Under Western Eyes: Feminist Scholarship and Colonial Discourses. Feminist Review 30 (autumn 1988).

Perkins Gilman, Charlotte (1911). The Man-made World: Or, Our Androcentric Culture. New York: Charlton Co.

Rubery, J, Moltó Carbonell, M.L. (coord.) (1993). Las mujeres y la recesión. Madrid: Ministerio de Trabajo y Seguridad Social.

Sales Gutiérrez, Laura i Camarasa i Casals, Mar. (2009). Dones en crisi. Barcelona: SURT. Fundació de Dones. Fundació Privada. Disponible en: http://www.surt.org/docs/publicats/2009/informe\%20recerca $\% 20$ dones $\% 20$ en $\%$ 20crisi.pdf

Valls, Francesc (2012). La pobreza femenina bajo el análisis (alternativo) del supuesto de autonomía individual. En: Ángel Belzunegui (coord.). Socialización de la pobreza en España. Barcelona: Icaria. 